\title{
Infrared spectrum and STM images of cyclohexene-2-ethanamine: First principle investigation
}

\author{
Tekin İzgi $^{a}$, Ethem Aktürk ${ }^{b, *}$, Oğuz Gülseren $^{c}$, Mustafa Şenyel $^{\mathrm{a}}$ \\ a Anadolu University, Department of Physics, Eskisehir, Turkey \\ ${ }^{\mathrm{b}}$ Hacettepe University, Department of Physics Engineering, Beytepe, 06800 Ankara, Turkey \\ ${ }^{\mathrm{c}}$ Bilkent University, Department of Physics, 06800 Ankara, Turkey
}

Received 9 August 2007; received in revised form 3 November 2007; accepted 8 November 2007

Available online 17 November 2007

\begin{abstract}
The vibrational properties of cyclohexene-2-ethanamine molecule are studied by both theoretically and experimentally. The theoretical calculations are performed using density functional perturbation theory (DFPT) based on plane wave pseudopotential method while the experimental technique is infrared (IR) spectroscopy. We also present the results of theoretical calculation of STM images for electron density of the cyclohexene-2-ethanamine. Our results also show that DFPT can be applied to the molecular systems in GGA approximation by comparing experimental and other commercial theoretical methods.
\end{abstract}

(C) 2007 Elsevier B.V. All rights reserved.

Keywords: Cyclohexene-2-ethanamine; Plane wave; IR spectrum; STM images; DFPT

\section{Introduction}

The design and synthesis of strong organic bases have long been an active field of research [1-4]. Infrared spectroscopy is a valuable tool in order to obtain information about the molecular structure and properties of the molecules. This technique is used widely in qualitative and quantitative molecular analysis. IR spectrum of interatomic vibrations can be used as structural probes for determining weak changes of structure or chemical bonding in molecules. Cyclohexene-2-ethanamine molecule consists of cyclohexene $\mathrm{C}_{6} \mathrm{H}_{10}$ group attached to the carbon of ethylamine $\left(\mathrm{C}_{2} \mathrm{H}_{7} \mathrm{~N}\right)$. There are previous works on the cyclohexene and ethylamine structures. Some studies showed that the lowest energy conformations of cyclohexene are in a half-chair form and a boat structure. Basically, the cyclohexene ring can interconvert from one twisted form to the other over the boat conformation with $C_{s}$ sym-

\footnotetext{
* Corresponding author.

E-mail address: eakturk@hacettepe.edu.tr (E. Aktürk).
}

metry [5]. The point symmetry group for trans-ethylamine ion is $C_{s}$ whereas there is no such symmetry for gauge-ethylamine [6]. Cyclohexene-2-ethanamine (CyHEA) has also important industrial applications, that is used as chemical intermediate in rubber industry. CyHEA was also used as a substrate and oxidizing agent for $\mathrm{Ru}$ complex. Sirimanne and May reported that dopamine $\beta$-monooxygenase (DBM) catalyzes stereo-selective allylic hydroxylation of CyHEA [7]. CyHEA was first synthesized by Izgi et al. [8] and some of IR and NMR properties of this compound were reported by them.

Density functional theory (DFT) is a widely used and very precise $a b$ initio technique which is used to calculate vibrational frequencies of organic compounds with great accuracy [12-17]. However, most calculations methods in the literature for molecular systems use B3LYB three parameter gradient corrected hybrid density functional theory method for all electronic structure and vibration properties with Gaussian software package. A previous theoretical investigation deal with dielectric and vibrational properties of amino acids by means of plane wave pseudopotential calculation [18]. There is a few study concerning 
the application of this method. The vibrational modes and STM images of this molecule have not been investigated by using plane wave pseudopotential calculation based on DFPT. The aim of the present study is to contribute to better understanding of plane wave pseudopotential calculation based on DFPT for molecular systems. The vibration properties are compared to experimental data and other theoretical method using the ab initio B3LYB method. The vibrational spectrum at $\Gamma$ point has been calculated both plane wave and B3LYB method. In this article we have carried out the vibration and STM image properties of solid-state phases of CyHEA. In Fig. 1, the geometry-optimized structure of CyHEA used in this study are shown.

\section{Computational methods}

In this paper, we use both the plane wave pseudopotential code PWSCF (Plane Wave Self Consistent Field) [10] and the B3LYB with 6-31G(d) method for all electronic structures with Gaussian03 [11] software package. The plane wave basis sets are complete and convergence may be improved by adjustment of kinetic energy cut-off [19]. We know that a plane wave basis set needs a periodic system. Molecules, atoms and clusters are investigated by using plane waves by means of a super cell approach (see in Fig. 2). In this method, the molecules is line in a unit cell of fictitious cell and this cell is sufficiently large enough to negligible interaction between neighboring cells. In this work, a cubic cell is used. The lattice constant of this cell is 20 bohr. In this case, it is sufficient to use $\Gamma$ point for carrying out integrations over Brillouin zone. In the present study, exchange and correlation effects have been included within both the generalized gradient approximation (GGA) and local density approximation (LDA). For all calculations, we have used Perdew-Zunger [21] and PerdewBurke-Ernzerhof [22] exchange-correlation parameterizations for LDA and GGA, respectively, and Vanderbilt [20] ultrasoft pseudopotentials. The pseudopotentials for $\mathrm{C}, \mathrm{N}, \mathrm{H}$ were derived by comparing available experimental data such as $\mathrm{C}-\mathrm{H}, \mathrm{N}-\mathrm{H}$ bond length and normal modes for $\mathrm{CH}_{4}$ and $\mathrm{NH}_{3}$ molecules, respectively. The electronic wave-

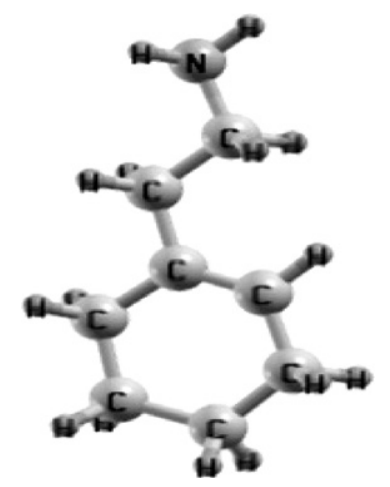

Fig. 1. The stable configuration of the cyclohexene-2-ethanamine.

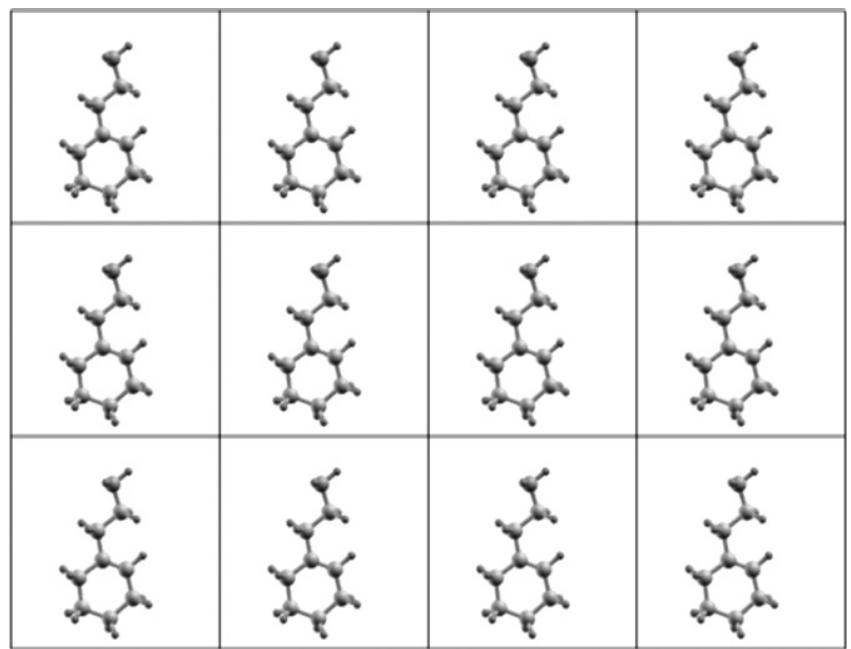

Fig. 2. A schematic illustration of a supercell geometry for a molecule.

functions were expanded in terms of plane waves with kinetic energy cut-off up to $25 \mathrm{Ry}$.

Earlier experimental investigations deal with the pure cyclohexene-2-ethanamine in liquid form which was obtained from Aldrich Chemical Co., USA and was used without further purification. The IR spectra of the molecule in liquid form was recorded in the range of 4000 $400 \mathrm{~cm}^{-1}$ using Perkin-Elmer FT-IR 2000 spectrometer with a resolution of $4 \mathrm{~cm}^{-1}$ [8].

\section{Results and discussion}

The calculated stable structure of CyHEA is shown in Fig. 1 which was drawn by XCrySDen (Crystalline Structures and Densities) program [9]. The vibrational assignments and frequencies of cyclohexene-2-ethanamine was reported experimentally by İzgi et al. [8]. The spectral properties of the molecule were evaluated through the calculated vibrational frequencies of the free ligand molecule. The calculated and experimental infrared spectra data of the molecule are given in Table 1. The experimental, GGA, LDA and B3LYB with 6-31G(d) results are also compared in Fig. 3.

The strong $\mathrm{N}-\mathrm{H}$ asymmetric and symmetric stretch bands seen in Table 1 are due to the contribution of ethylamine (see Fig. 4). C-H stretch bands between 3000 and $3100 \mathrm{~cm}^{-1}$ are attributed to cyclohexene group and the very strong $\mathrm{C}-\mathrm{H}$ stretch bands at 2926 and $2835 \mathrm{~cm}^{-1}$ result from ethylamine. The very strong bands are attributed to the attachment of ethylamine and cyclohexene and appear between 2830 and $2920 \mathrm{~cm}^{-1}$. Most of the modes below the $1300 \mathrm{~cm}^{-1}$ arise from cyclohexene. If the vibrational assignments of the molecule involving these groups are investigated, it is seen that the assignments obtained for the molecule also involve the group frequencies. Furthermore, the observed medium broad band appears at $829 \mathrm{~cm}^{-1}$ is an $\mathrm{N}-\mathrm{H}$ bending band as well as a group frequency. There is also a good agreement between the experimental and the 
Table 1

Experimental and theoretical values of vibration frequencies of cyclohexene-2-ethanamine

\begin{tabular}{|c|c|c|c|c|c|}
\hline \multirow[t]{2}{*}{ Mode } & \multicolumn{2}{|l|}{ Experimental [8] } & \multicolumn{3}{|c|}{ Calculated } \\
\hline & Assignments & IR freq. & LDA & GGA & $\begin{array}{l}\text { B3LYB with } \\
\text { 6-31G(d) }\end{array}$ \\
\hline$v_{1}$ & $N-H a$-str & $3366 \mathrm{~s}$ & 3447 & 3436 & 3394 \\
\hline$v_{2}$ & $N-H s$-str & $3288 \mathrm{~s}$ & 3353 & 3347 & 3276 \\
\hline$v_{3}$ & $v_{23}$ & $3097 \mathrm{vw}$ & 3015 & 3045 & 3090 \\
\hline$v_{4}$ & $v_{1}$ & $3043 \mathrm{~m}$ & 2979 & 2998 & 3044 \\
\hline$v_{5}$ & $\mathrm{C}-\mathrm{H} \operatorname{str}\left(\mathrm{CH}_{3}\right)$ & $2995 \mathrm{~m}$ & 2954 & 2971 & 2996 \\
\hline$v_{6}$ & $v_{2}+\mathrm{C}-\mathrm{H} \operatorname{str}\left(\mathrm{CH}_{3}\right)$ & $2926 v s$ & 2925 & 2921 & 2918 \\
\hline$v_{7}$ & $v_{25}+\mathrm{C}-\mathrm{H} \operatorname{str}\left(\mathrm{CH}_{2}\right)$ & $2894 \mathrm{vw}$ & 2896 & 2899 & 2898 \\
\hline$v_{8}$ & $v_{26}$ & $2877 \mathrm{vw}$ & 2880 & 2897 & 2881 \\
\hline$v_{9}$ & $v_{27}+\mathrm{C}-\mathrm{H} \operatorname{str}\left(\mathrm{CH}_{3}\right)$ & $2857 \mathrm{vs}$ & 2856 & 2879 & 2858 \\
\hline$v_{10}$ & $v_{5}+\mathrm{C}-\mathrm{H} \operatorname{str}\left(\mathrm{CH}_{3}\right)$ & $2836 v s$ & 2827 & 2857 & 2840 \\
\hline$v_{11}$ & $v_{6}$ & $1666 \mathrm{~m}$ & 1712 & 1693 & 1660 \\
\hline$v_{12}$ & $\mathrm{NH}_{2}$ sciss & $1600 \mathrm{mb}$ & 1562 & 1594 & 1610 \\
\hline$v_{13}$ & $\mathrm{CH}_{2}$ sciss & $1505 \mathrm{vw}$ & - & - & 1514 \\
\hline$v_{14}$ & $\mathrm{C}-\mathrm{H}$ bend $\left(\mathrm{CH}_{3}\right)$ & $1473 \mathrm{vw}$ & - & 1464 & 1479 \\
\hline$v_{15}$ & $v_{28}+\mathrm{C}-\mathrm{H}$ bend $\left(\mathrm{CH}_{3}\right)$ & $1448 \mathrm{vw}$ & 1439 & 1442 & 1447 \\
\hline$v_{16}$ & $v_{8}$ & $1438 \mathrm{~s}$ & 1431 & 1425 & 1428 \\
\hline$v_{17}$ & $\mathrm{CH}_{2}$ wag & $1384 \mathrm{w}$ & 1391 & 1384 & 1387 \\
\hline$v_{18}$ & $v_{9}+\mathrm{C}-\mathrm{H}$ bend $\left(\mathrm{CH}_{3}\right)$ & $1370 \mathrm{vw}$ & 1376 & 1359 & 1372 \\
\hline$v_{19}$ & $v_{10}$ & $1344 \mathrm{~m}$ & 1352 & 1338 & 1351 \\
\hline$v_{20}$ & $v_{30}$ & $1334 \mathrm{w}$ & 1328 & 1334 & 1340 \\
\hline$v_{21}$ & $\mathrm{NH}_{2}$ twist & $1307 \mathrm{w}$ & 1320 & 1309 & 1309 \\
\hline$v_{22}$ & $v_{32}$ & $1269 \mathrm{~m}$ & 1285 & 1298 & 1271 \\
\hline$v_{23}$ & $v_{11}+\mathrm{CH}_{2}$ twist & $1242 \mathrm{w}$ & 1248 & 1247 & 1228 \\
\hline$v_{24}$ & $v_{12}$ & $1215 \mathrm{w}$ & 1212 & 1227 & 1211 \\
\hline$v_{25}$ & $v_{34}$ & $1136 \mathrm{~m}$ & 1131 & 1139 & 1124 \\
\hline$v_{26}$ & $(C-C, C-N) a-\operatorname{str}$ & $1086 \mathrm{w}$ & 1101 & 1092 & 1079 \\
\hline$v_{27}$ & $v_{15}$ & $1066 \mathrm{w}$ & - & 1080 & 1067 \\
\hline$v_{28}$ & $v_{35}$ & $1049 \mathrm{w}$ & 1045 & 1042 & 1051 \\
\hline$v_{29}$ & $v_{36}+\mathrm{CH}_{3}$ rock & $1022 \mathrm{w}$ & 1014 & 1023 & 1022 \\
\hline$v_{30}$ & $v_{16}$ & $966 \mathrm{w}$ & 976 & 975 & 973 \\
\hline$v_{31}$ & $v_{37}$ & $919 \mathrm{~m}$ & 921 & 920 & 937 \\
\hline$v_{32}$ & $v_{17}$ & $906 \mathrm{vw}$ & 919 & 903 & 925 \\
\hline$v_{33}$ & $v_{38}$ & $857 \mathrm{w}$ & 834 & 843 & 862 \\
\hline$v_{34}$ & $v_{18}+\mathrm{CH}_{2}$ rock & $829 \mathrm{~m}$ & 824 & 818 & 822 \\
\hline$v_{35}$ & $v_{19} \mathrm{NH}_{2}$ wag & $800 \mathrm{~m}$ & - & 804 & 808 \\
\hline$v_{36}$ & $v_{39}$ & $720 \mathrm{sh}$ & 733 & 734 & 730 \\
\hline$v_{37}$ & $v_{40}$ & $647 w$ & 680 & 686 & 664 \\
\hline$v_{38}$ & $v_{20}$ & $497 \mathrm{vw}$ & 518 & 512 & 515 \\
\hline$v_{39}$ & $v_{41}$ & $448 w$ & 417 & 411 & 450 \\
\hline
\end{tabular}

Ass., assignments; Exp., experimental; Freq., frequency; v, very; s, strong; $\mathrm{m}$, medium; w, weak, sh, shoulder; b, broad; str, stretching; bend, bending; sciss, scissoring; twist, twisting; wag, wagging; s, symmetric; a, asymmetric.

theoretical vibrational frequencies in the region of 4000 $400 \mathrm{~cm}^{-1}$ except some GGA, LDA and B3LYB results. It is seen in Table 1, the present calculation gives four vibration modes for LDA and one vibration mode for GGA that could not be founded theoretically. These invisible modes in calculation is due to both systematic errors in the first principle calculation and to the fact that the experimental results belong to liquid phase and theoretical calculations have solid phase. On the other hand, the experimental values of modes are measured at room temperature but theoretical results are at zero temperature. While B3LYB with $6-31 \mathrm{G}(\mathrm{d})$ provides a very satisfying results, LDA and GGA gives somewhat larger deviations.

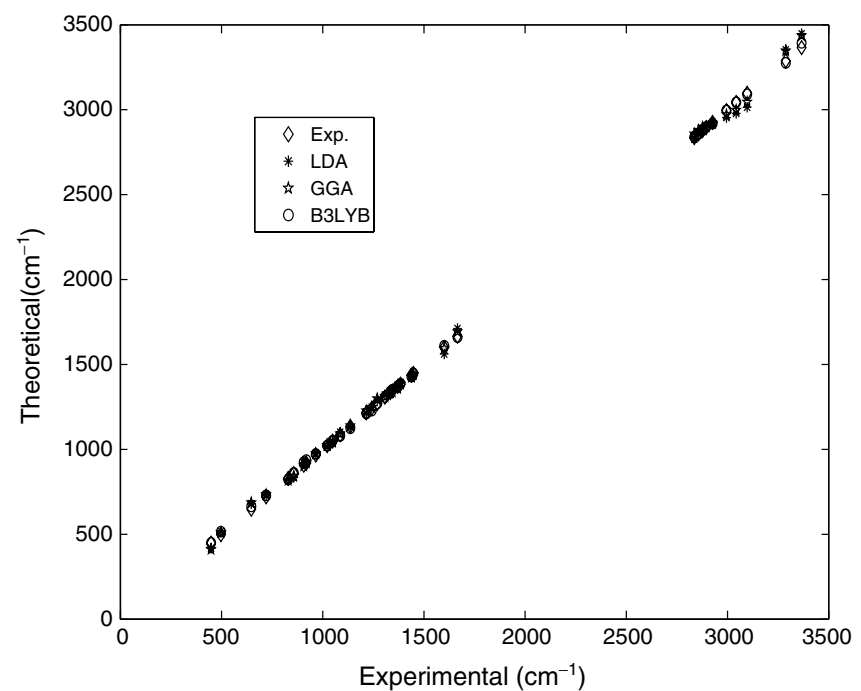

Fig. 3. The comparison of experimental data with GGA, LDA and B3LYB results.
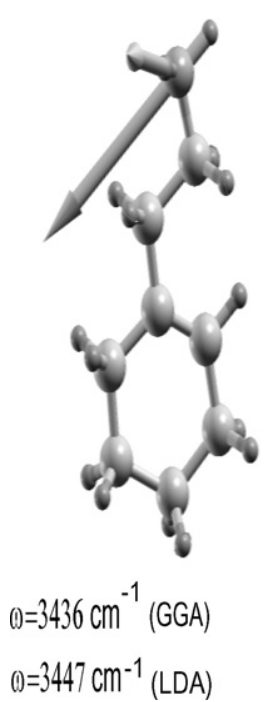
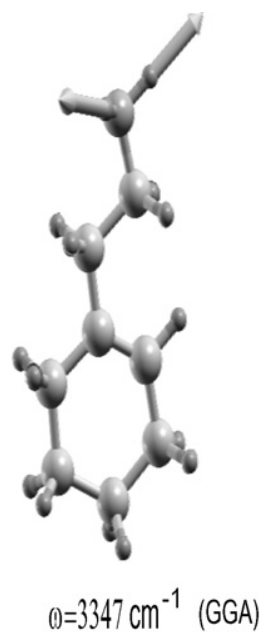

$0=3353 \mathrm{~cm}^{-1}$ (LDA)
Fig. 4. Calculated $\mathrm{N}-\mathrm{H}$ asymmetric and symmetric stretch.

However, our results shows that GGA is significantly better than the LDA for molecules.

Finally, we examined the electronic properties by using calculated STM images for cyclohexene-2-ethanamine. In Figs. 5 and 6 which were drawn by using XCrySDen, we calculated the STM images at constant current and bias voltage -2.5 and $2.5 \mathrm{eV}$, respectively. STM images does not only show geometric structure but it is also described by the electronic structure of sample. In generally, STM images is very important for investigation of adsorption of molecules on surface. However, STM is a powerful tool to image molecules at atomic scale. It is seen in Fig. 5, cyclohexene-2-ethanamine has only one $C=C$ which is constructed two $\mathrm{sp}^{2}$ hybridized carbons atoms with a pair of electrons in the $\pi$-molecular orbital. There is also a depletion of charge in core region. Each carbon contributes $\mathrm{p}$ orbital and five $p$ orbital overlap to give cyclic $\pi$ system. The electron density are not founded by one carbon atom 


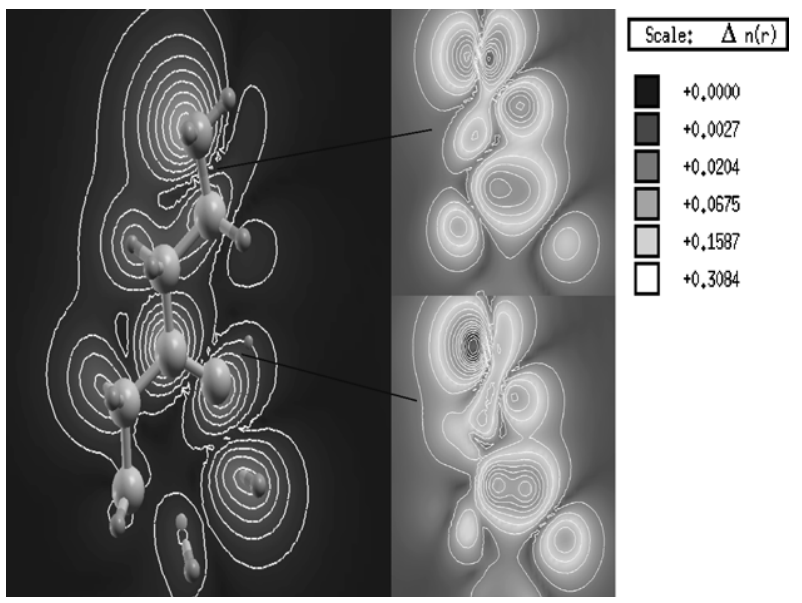

Fig. 5. Calculated STM images for HOMO.

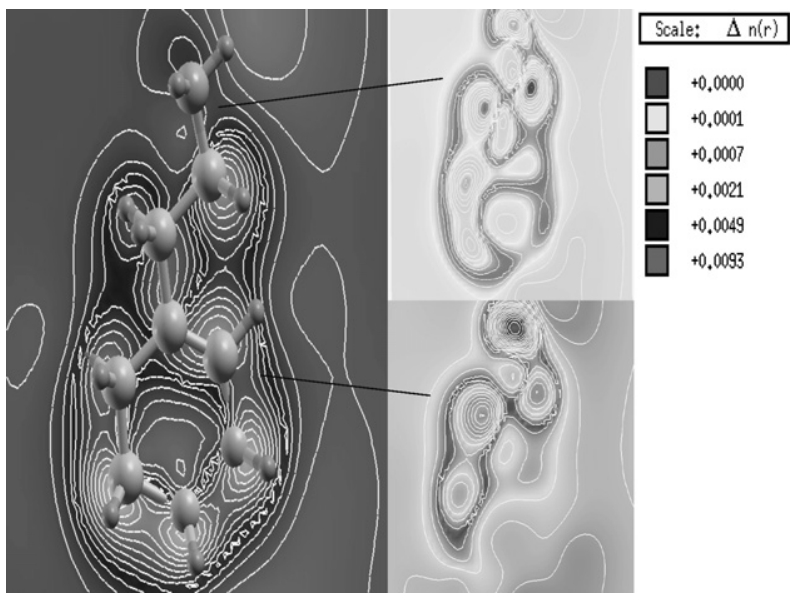

Fig. 6. Calculated STM images for LUMO.

or another, the electron density are placed in bonding region and environment. We can see theoretically that molecules are bonding with accumulation of electrons between bounded atoms. The HOMO region is also smaller to butterfly images. In Fig. 6, the LUMO level which arises from the p-type orbitals of eight carbon atoms. This level is almost empty but only near the nitrogen atom has smaller electron density.

On the other hand, the highest occupied molecular orbital (HOMO) and lowest unoccupied molecular orbital (LUMO) play an important role in governing site selection in chemical reactions and his frontier electron theory are observed by Fukui [23]. The energy difference between HOMO and LUMO is called as the HOMO-LUMO gap. In this calculation, we obtained that HOMO and LUMO level are -4.402 and $-0.399 \mathrm{eV}$, respectively. Hence, HOMO-LUMO gap for cyclohexene-2-ethanamine is $4.003 \mathrm{eV}$. Thus, we can say that this molecules is used as reactant.

\section{Conclusion}

The vibrational modes of CyHEA molecule have calculated using different theoretical methods. In comparison with vibrational modes of cyclohexene-2-ethanamine obtained from FT-IR, the present calculations are good agreement with measurements for some vibration modes. Any inconsistency noted between the observed and the calculated vibrational modes using DFPT may be due to the fact that have a molecule in a solid phase. Thus, density functional perturbation theory (DFPT) methods are suitable for the calculation of ground state properties and potential energies. Hence, DFPT is an excellent method for calculating vibrational spectra and STM images from first principles.

\section{References}

[1] F. Hibbert, Acc. Chem. Res. 17 (1984) 115.

[2] H.A. Staab, T. Saupe, Angew. Chem. 100 (1988) 895; H.A. Staab, T. Saupe, Angew. Chem. Int. Ed. Engl. 27 (1988) 865.

[3] R.W. Alder, Chem. Rev. 89 (1989) 1215.

[4] R.W. Alder, Tetrahedron 46 (1990) 683.

[5] S. Rodin-Bercion, L. Lespade, D. Cavagnat, J.C. Cornut, J. Mol. Struct. (Theochem) 526 (2000) 343.

[6] D. Zeroka, J.O. Jensen, A.C. Samuels, J. Mol. Struct. (Theochem) 465 (1999) 119.

[7] S.R. Sirimanne, S.W. May, J. Am. Chem. Soc. 110 (1988) 7560.

[8] T. İzgi, Ö. Alver, C. Parlak, M.T. Aytekin, M. Şenyel, Spectrochem. Acta A: Mol. Biomol. Spectrosc. 68 (1) (2007) 55.

[9] A. Kokalj, Comp. Mat. Sci. 28 (2003) 155. <www.xcrysden.org/>.

[10] S. Baroni, A. Dal Corso, S. de Gironcoli, P. Giannozzi, <http:// www.sissa.it/cm/PWcodes $>$..

[11] M.J. Frisch et al., Gaussian 03, Inc., Pittsburg, PA, 1998.

[12] N.C. Handy, P.E. Maslen, R.D. Amos, J.S. Andrews, C.W. Murray, G.J. Laming, Chem. Phys. Lett. 197 (1992) 506.

[13] N.C. Handy, C.W. Murray, R.D. Amos, J. Phys. Chem. 97 (1993) 4392.

[14] P.J. Stephens, F.J. Devlin, C.F. Chabalowski, M.J. Frisch, J. Phys. Chem. 98 (1994) 11623.

[15] F.J. Devlin, J.W. Finley, P.J. Stephens, M.J. Frisch, J. Phys. Chem. 99 (1995) 16883.

[16] S.Y. Lee, B.H. Boo, Bull. Korean Chem. Soc. 17 (1996) 754.

[17] S.Y. Lee, B.H. Boo, Bull. Korean Chem. Soc. 17 (1996) 760.

[18] P.R. Tulip, S.J. Clark, Phys. Rev. B 74 (2006) 064301.

[19] P.R. Tulip, S.J. Clark, J. Chem. Phys. 121 (2004) 5201.

[20] D. Vanderbilt, Phys. Rev. B 41 (1990) 7892.

[21] J.P. Perdew, A. Zunger, Phys. Rev. B 23 (1981) 5048.

[22] J.P. Perdew, K. Burke, M. Ernzerhof, Phys. Rev. Lett. 77 (1996) 3865.

[23] K. Fukui, T. Yonezawa, H. Shingu, J. Chem. Phys. 20 (1952) 722. 